This item was submitted to Loughborough's Research Repository by the author.

Items in Figshare are protected by copyright, with all rights reserved, unless otherwise indicated.

\title{
Relations between parameters of fracture processes on different scale levels
}

PLEASE CITE THE PUBLISHED VERSION

https://doi.org/10.1134/S1028335818110095

\section{PUBLISHER}

(c) Pleiades Publishing, Ltd., Published by Springer

VERSION

AM (Accepted Manuscript)

\section{PUBLISHER STATEMENT}

This work is made available according to the conditions of the Creative Commons Attribution-NonCommercialNoDerivatives 4.0 International (CC BY-NC-ND 4.0) licence. Full details of this licence are available at: https://creativecommons.org/licenses/by-nc-nd/4.0/

\section{LICENCE}

CC BY-NC-ND 4.0

\section{REPOSITORY RECORD}

Mikhailova, N.V., G. A. Volkov, Yu. V. Petrov, I. V. Smirnov, P.Y. Onawumi, Anish Roy, and Vadim Silberschmidt. 2019. "Relations Between Parameters of Fracture Processes on Different Scale Levels". figshare. https://hdl.handle.net/2134/36529. 


\title{
Relations between Parameters of Fracture Processes on Different Scale Levels
}

\author{
N. V. Mikhailova ${ }^{a, *}$, G. A. Volkov ${ }^{a}$, Corresponding Member of the RAS Yu. V. Petrov ${ }^{a, b}$, \\ I. V. Smirnov ${ }^{a}$, P. Onawumi ${ }^{c}$, A. Roy ${ }^{c}$, and V. Silberschmidt ${ }^{c}$
}

\begin{abstract}
The processes of ultrasonically-assisted drilling (UAD) and the dynamic tests on split Hopkinson pressure bar (SHPB), fracture in which is implemented at various structural-scale levels, are considered. The simulation of UAD based on the Hertz contact problem and the structural-time criterion is presented. The problem of using the value of the fracture incubation time and its linear size obtained from the tests on SHPB in the simulation is considered. A principle of equal power is used for converting the strength parameters into another structural-scale level. The theoretical curve obtained in the simulation is compared with the results of experiments on conventional drilling (CD) and UAD.
\end{abstract}

DOI: $10.1134 / \mathrm{S} 1028335818110095$

When solving the problems of fracture mechanics, it is necessary first of all to set the key features that determine the fracture event itself, corresponding to specific tests, for example, to consider the formation of microcracks in a sample or its macroscopic fragmentation as the fracture thereby implicitly determining the representative volume of the fracture. Thus, depending on the type of loading action, fracture can occur at different scale levels [1,2]. The scale on which the fracture is implemented determines the values of the strength parameters of a material or the mechanical characteristics critical for this level.

A convenient tool that makes it possible to specify the relationship between the characteristics obtained at different structural-scale levels is the principle of equal power [3]. The study shows the consistency of the principle of equal power with the experimental results obtained for various processes associated with fracture: ultrasonically-assisted drilling (UAD) [4, 5] and the dynamic tests on split Hopkinson pressure bar (SHPB) [7]. As a test material, fine-grained marble was used. The fracture in these tests is implemented under loadings of various intensity, as a result of which the material is damaged at different scales. Analysis of the experimental data showed that it is impossible to

\footnotetext{
${ }^{a}$ St. Petersburg State University, St. Petersburg, 199034 Russia

${ }^{b}$ Institute for Problems in Mechanical Engineering, Russian Academy of Sciences, St. Petersburg, 199178 Russia ${ }^{c}$ Loughborough University, Loughborough, LE11 $3 T U$ United Kingdom

*e-mail: natasha-mikh@yandex.ru
}

use the same values of the strength parameters for the two types of fracture. Thus, in order to simulate the process correctly, it is of importance to know the critical characteristics corresponding to different scale levels, as well as the possibility of recalculating their values when passing from one scale to another.

During UAD, on the core drill bit with diamond coating ultrasonic vibrations are imposed which under certain amplitude-frequency modes initiate the impact interaction of diamond particles with the material surface. The corresponding analytical simulation of UAD can be constructed on the basis of solution of the Hertz contact problem on the impact of an absolutely solid particle on a deformable half-space [8]. For determining the fracture condition upon particle impact on the material, the structural-time criterion [9] is used, the parameters of which are the fracture incubation time $\tau$ and the static strength $\sigma_{\mathrm{c}}$ of the material:

$$
\max _{t} \int_{t-\tau}^{t} \sigma(s) d s \leq \tau \sigma_{\mathrm{c}} .
$$

It was shown in $[4,11]$ that the kinetic energy of a particle within the framework of this model is calculated as follows:

$$
K=\alpha \frac{t_{0}^{3} \sigma_{\max }^{13 / 2}}{\rho^{3 / 2} E^{4}},
$$

where $t_{0}$ is the contact time of the particle in one cycle, $\sigma_{\max }$ is the amplitude of the tensile stresses, $\rho$ is a parameter having the density dimension that determines the intensity of the grinding process, $\alpha$ is a constant, and $E$ is the elasticity modulus. Further, the 
Table 1. Incubation time $\tau$ and the linear size $d$ at two fracture scale levels: I, dynamic tests at the SHPB; and II, drilling with ultrasound

\begin{tabular}{c|r|c}
\hline Fracture level & $\tau, \mu \mathrm{s}$ & $d, \mathrm{~mm}$ \\
\hline I & 79 & 6.4 \\
II & 1 & 0.4 \\
\hline
\end{tabular}

energy $K$ and the linear size $d$ are used for simulating the cutting force $F$, which is measured during the UAD tests [10]:

$$
F=K / d .
$$

The value of the fracture incubation time necessary for further calculations is determined from the dynamic experiments at the SHPB [7]: the dynamic curve is constructed from the test results and enables us to find the desired parameter on the basis of criterion (1). The linear size $d$ is calculated through the strength parameters and the critical fracture toughness: $d=\frac{2 K_{I c}^{2}}{\pi \sigma_{\mathrm{c}}^{2}}$. The parameters $\tau$ and $d$ calculated in this way, nevertheless, do not allow us to obtain an adequate structural-time model of UAD. During the dynamic tests on SHPB, a marble sample was under impact pulses the intensity and duration of which were much higher than that in the experiments on UAD. The sizes of the fracture regions in the two types of tests differed by orders of magnitude; i.e., the two types of fracture considered were implemented at different scale levels to which different space-time elementary (representative) volumes correspond [2]. Thus, there is a fundamental problem of determining

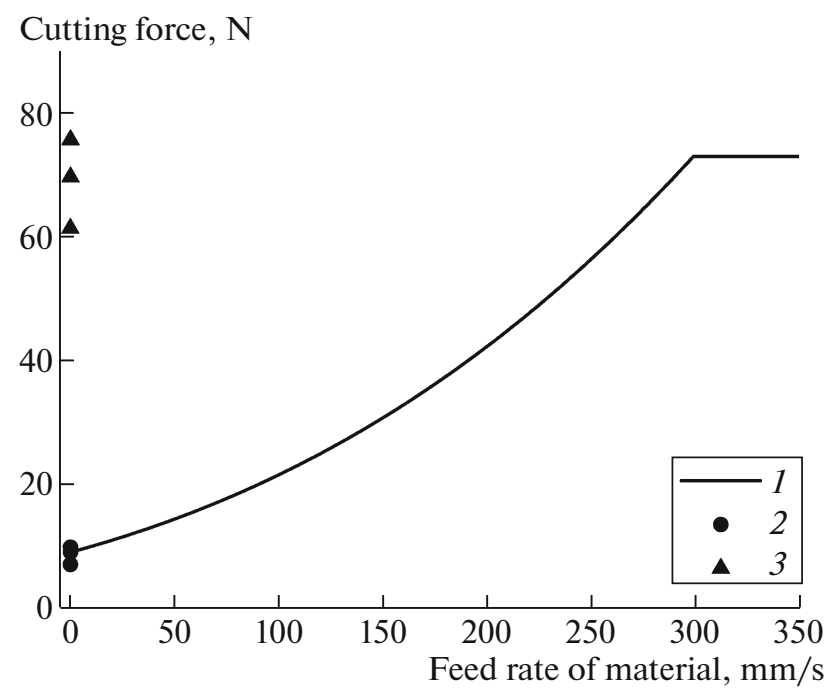

Fig. 1. Dependence of the cutting force on the feed rate of the material: (1) theoretical calculation; (2) experimental data on ultrasonically assisted drilling; and (3) experimental data on conventional drilling. the values of the parameters $d$ and $\tau$ for the fracture scale level on which the ultrasonic drilling is implemented.

The principle of equal power [3] is based on the constancy of the average power for each structural-scale level. The average power is determined by the ratio of the energy spent on the fracture of an elementary spatial cell at a given scale level to the corresponding value for the same incubation time. In this case, the principle of equal power for two structural-scale levels can be represented as follows:

$$
\frac{Q_{1}}{\tau_{1}}=\frac{Q_{2}}{\tau_{2}}=\text { const. }
$$

The fracture energy $Q_{1}$ during the dynamic tests on the SHPB is calculated as the strain energy under threshold loading conditions:

$$
Q_{1}=\frac{1}{2} E \varepsilon^{2} V
$$

where the volume $V$ is taken as the volume of the part of the loaded bar that encloses the impact pulse along the length and the deformation is calculated as $\varepsilon=$ $\max \left(\varepsilon_{i}-\varepsilon_{r}-\varepsilon_{t}\right)$, where $\varepsilon_{i}, \varepsilon_{t}$, and $\varepsilon_{r}$ are the deformations of the incident, transmitted, and reflected pulses.

Under UAD, the energy $Q_{2}$ is the fracture threshold energy $K$ of the material. Thus, using the principle of equal power (3), we can determine the incubation time $\tau_{2}$ for the UAD process by the following relationship:

$$
\tau_{2}=\frac{\tau_{1} K}{Q_{1}}
$$

It is assumed that the linear size $d$ for UAD is equal to the average diameter of the diamond particles. The value of the parameters under consideration for each fracture scale level is presented in Table 1.

The obtained value of $\tau_{2}$ from the principle of equal power (4) agrees with the assumption about the relation of two scale levels through the incubation time [2]:

$$
\tau_{2}=\frac{d_{1}}{c}=1.65 \mu \mathrm{s}
$$

Using the parameters obtained, we simulated the dependence of cutting force (2) on the feed rate of the material. Figure 1 shows the comparison of the theoretical curve with the data obtained in the tests for ultrasonically assisted and conventional drilling. The increase in forces obtained in the graph is caused by the fact that the contact time of a particle with the surface increases with the feed rate of the material, and the contact becomes constant if a certain critical speed is achieved. Thus, the drilling mode changes from ultrasonic to conventional. However, the technical parameters of the experimental installation prevent from testing over the entire speed range. 
Thus, two processes were considered in which fractures are occurred at different structural-scale levels. When a diamond particle strikes the surface during UAD, a much smaller volume of the material is destroyed than under the impact actions on the SHPB at which the tests were carried out for determining the marble strength characteristics, including the incubation time. This parameter is necessary for simulating the UAD; however, due to the fact that the process under consideration takes place on a different scale, the use of the values of the structure-time characteristics obtained in the tests with the SHPB is not correct. The principle of equal power made it possible to relate two structural-scale levels and to obtain the value of the desired parameter of the UAD model, which is in good agreement with the experimental data.

\section{ACKNOWLEDGMENTS}

This study was supported by the Marie Curie Foundation (FP7 IRSES project TAMER IRSESGA-2013-610547) and by the Russian Foundation for Basic Research (project no. 17-01-00618) and by grant of the President of the Russian Federation (project no. MK-6312.2018.1).

\section{REFERENCES}

1. V. E. Panin, V. A. Likhachev, and Yu. V. Grinyaev, Structural Levels of Deformation in Solids (Nauka, Novosibirsk, 1985) [in Russian].

2. Y. V. Petrov, A. A. Gruzdkov, and V. A. Bratov, Phys. Mesomech. 15, 232 (2012).

3. Yu. V. Petrov, A. A. Gruzdkov, and N. F. Morozov, Dokl. Phys. 50 (9), 448 (2005).

4. N. A. Gorbushin, G. A. Volkov, and Yu. V. Petrov, Tech. Phys. 52 (6), 852 (2014).

5. P. N. H. Thomas and V. Babitsky, J. Sound and Vibration 308, 815 (2007).

6. Yuri V. Petrov, Ivan V. Smirnov, Grigory A. Volkov, and Andrei K. Abramian, J. Rock Mech. and Geotechn. Eng. 9, 125 (2017).

7. H. Kolsky, Proc. Phys. Soc. 62 (11), 676 (1949).

8. K. Johnson, The Mechanics of Contact Interaction (Nauka, Moscow, 1989) [in Russian].

9. N. F. Morozov and Yu. V. Petrov, Problems of Dynamics of Fracture in Solids (SPbGU, St. Petersburg, 1997) [in Russian].

10. G. A. Volkov, V. A. Bratov, A. A. Gruzdkov, V. I. Babitsky, Y. V. Petrov, and V. V. Silberschmidt, Shock Vibr. Dig. 18, 333 (2011).

11. G. A. Volkov, N. A. Gorbushin, and Yu. V. Petrov, Mech. Solids 47 (5), 491 (2012). 